\title{
Déchets techniques et réglementation
}

Jean-Louis Millo

\section{(2) OpenEdition \\ Journals}

Édition électronique

URL : http://journals.openedition.org/plc/529

DOI : $10.4000 /$ plc.529

ISSN : 2117-5209

\section{Éditeur}

L'Harmattan

\section{Édition imprimée}

Date de publication : 1 janvier 1999

Pagination : 281-289

ISSN : 1279-8657

\section{Référence électronique}

Jean-Louis Millo, « Déchets techniques et réglementation», Pouvoirs dans la Caraïbe [En ligne], 11 |

1999, mis en ligne le 08 mars 2011, consulté le 10 décembre 2020. URL : http://

journals.openedition.org/plc/529; DOI : https://doi.org/10.4000/plc.529 


\section{Déchets techniques et réglementation}

\section{EN GUISE D'INTRODUCTION : QUELQUES EVIDENCES}

Tout d'abord, le déchet n'est pas une fatalité : depuis toujours, nous avons vécu avec nos produits «ultimes »; par exemple, nos grands-parents issus de milieux ruraux, traitaient leurs ordures ménagères en triant ce qui brûlait (régulièrement, on allumait un feu au fond du jardin) et ce qui se dégradait (un tas de compost voisinait souvent avec la place à feu).

Depuis l'avènement de la société de consommation, basée sur la production de masse standardisée, ... et sur l'emballage, la question de déchets a pris une acuité particulière, renforcée par la transformation de l'habitat (urbain) et des modes de vie (loisirs, spécialisation du travail...).

Ainsi, apparaissent progressivement de véritables politiques de gestion des déchets, nécessitant des règles claires, des textes réglementaires; il est frappant de constater qu'en France, le texte de base dans le domaine de l'eau est la loi sur l'eau de 1964, alors que sur les déchets, il a fallu attendre la loi de juillet 1992 pour voir préciser clairement les principes de la politique nationale en matière de gestion des déchets ; presque 30 ans après ! 


\section{Qu'est ce qu'un déchet?}

La définition intuitive est généralement donnée par rapport à l'usage d'un produit, d'un matériau... dont on ne sait plus quoi faire, et dont il faut se débarrasser : toutefois, l'usage peut être terminé chez certains et pas chez d'autres: nous jetons nos boites de bière en aluminium, les Africains les récupèrent pour en faire des timbales, des objets courants...

La notion de déchets est donc liée à la durée de vie d'un produit, et ce produit dont l'usage est terminé, peut être lui-même recyclé ou transformé en sous produits réutilisables.

Deux définitions complémentaires apparaissent :

Le recyclage : on parle plutôt aujourd'hui de valorisation des déchets, avec une valorisation dite «matière » lorsque tout ou partie du produit est récupéré et fait l'objet d'un nouvel usage, et une valorisation «énergétique » lorsque le déchet produit de l'énergie (chaleur, électricité),

Le déchet ultime : c'est le stade où «dans les conditions techniques et économiques du moment » on ne trouve plus d'usage au déchet; il faut donc le détruire, le stocker, l'exporter... Ce déchet ultime n'est pas forcément le même dans différentes conditions économiques : l'insularité peut modifier par exemple le niveau de récupération : la bouteille en verre est actuellement un déchet ultime en Martinique.

Atteindre le déchet ultime peut donc devenir l'objectif d'une politique de gestion. On remarquera que cette notion évolue dans le temps : les déchets radioactifs sont aujourd'hui stockés dans l'attente d'un éventuel traitement dans les années futures. Autre remarque, le recyclage de déchets devient une activité économique à part entière.

Une autre approche du déchet est sa classification :

Par catégories physiques : déchets liquides et solides, radioactifs ou non, déchets ferreux, chimiques, organiques, ... Une grande partie de nos déchets liquides n'entre pas dans la catégorie des déchets, ce sont les eaux 
usées urbaines et industrielles qui participent au cycle de l'eau : par exemple, une eau d'égout est considérée comme un rejet aqueux dans le milieu naturel, elle peut être épurée (valorisation) et le terme de déchet ne réapparaît que lorsque l'on a piégé un déchet solide, les boues de stations d'épuration !

Par producteur de déchet : le déchet peut être issu de l'activité d'une famille, c'est alors une ordure ménagère, par l'activité d'un industriel, déchet industriel ou par l'activité des hôpitaux, médecins, infirmiers...

Réglementairement, on distinguera :

- les ordures ménagères,

- les déchets industriels banals,

- les déchets industriels spéciaux,

- les déchets contaminés ou déchets de soins.

\section{Les principes de la loi du 13 Juillet 1992 \\ Premier principe : «pollueur-payeur»}

La loi stipule que le producteur d'un déchet doit payer le prix de l'élimination de ce déchet; ainsi, le citoyen qui jette ses emballages, même fournis par l'industrie agroalimentaire, est responsable de l'élimination. Cette élimination comprend en général la collecte puis le traitement du déchet; ce traitement peut être avec ou sans valorisation.

Le citoyen se regroupe au sein de collectivités pour leur confier le soin d'éliminer les ordures ménagères : communes, syndicats de communes... Il faut toutefois noter que si une filière n'est pas organisée au niveau des collectivités, le citoyen reste responsable : c'est le cas de la voiture usagée : tout automobiliste est responsable de l'élimination de sa vieille carcasse, c'est encore plus évident avec la vieille batterie.

De même, l'industriel est responsable de l'élimination de ses déchets, et l'agriculteur, des siens; exemple intéressant à analyser, les gaines bleues de la culture de banane. Ces gaines commencent à être collectées dans des bacs 
analogues à ceux des ordures ménagères, pourtant, ces gaines ne doivent pas être mélangées aux ordures (dans l'incinérateur, par exemple), sans que le coût de leur traitement ait été individualisé, pour le mettre à la charge du producteur de banane.

\section{Deuxième principe : répartir les responsabilités}

Afin d'organiser les différentes filières de collecte et de traitement des déchets, l'Etat a la responsabilité de mettre en place une planification par catégories de déchets, les collectivités et les différents producteurs de déchets ont la responsabilité de mettre en place les dispositifs de collecte et de traitement et de les faire fonctionner.

Ainsi, trois types de plans ont été créés :

- le plan départemental de collecte et de traitement des ordures ménagères, sous la responsabilité du Préfet de Département (DAF/ADEME),

- le plan régional pour les déchets industriels spéciaux (PREDIS), sous la responsabilité du Préfet de Région (DRIRE),

- le Schéma Régional de collecte et traitement des déchets de soins, sous la responsabilité du Préfet de Région (DRASS),

Les différents plans fixent des échéances de réalisation des équipements et donnent des coûts d'objectifs ; pour les ordures ménagères, il est fixé au niveau national que les décharges non autorisées devront disparaître en 2002.

En Martinique, le plan «ordures ménagères » est adopté depuis janvier 1997, les deux autres sont en fin d'élaboration. 


\section{Troisième principe : affirmer la valeur économique du déchet}

Dès le stade de la conception d'un produit, les impacts des déchets engendrés doivent être pris en compte ; ainsi, pour les emballages, un système de mutualisation a été mis en place (éco-emballage) afin de subventionner les initiatives des collectivités visant le tri des emballages et leur recyclage.

Les grands groupes privés se sont déjà reconvertis dans les filières de collecte et de traitement des ordures ménagères; les filières qui privilégient le tri et une valorisation «matière » sont évidemment des secteurs fortement créateurs d'emplois.

Le déchet a également une forte implication économique pour son producteur : par exemple, comment est payée la contribution des ménages ?

La réglementation en matière de financement des coûts récurrents dans le secteur des déchets ménagers est fondamentalement différente de celle du secteur de l'eau :

- les coûts de production et de dépollution des eaux sont financés au coût réel par le paiement d'un «service de l'eau » (vérité des prix) : le détail du prix de l'eau et des différents prélèvements apparaît sur la facture d'eau ;

- par contre, la collecte et le traitement des déchets sont financés par la «taxe d'ordure ménagère », véritable impôt local au même titre que la taxe d'habitation ou la taxe foncière; l'assiette de la taxe d'ordure ménagère est d'ailleurs la même que celle de la taxe foncière.

Le résultat est que la contribution du citoyen n'est pas forcément proportionnelle au coût du service : une habitation éloignée est desservie de la même façon, par contre, les budgets des collectivités, contrairement à l'eau, peuvent «subventionner» les services d'ordures ménagères. Pour avoir un ordre de grandeur en tête, on retiendra qu'un habitant peut produire annuellement jusqu'à une tonne d'ordures ménagère et que la tonne collectée et traitée peut coûter de 600 F/T à 1200 F/T. 


\section{Qu'en est-il à la Martinique?}

Les filières industrielles et déchets de soin sont très en retard sur les plans qui vont être adoptés: par exemple, les incinérateurs de déchets contaminés ne sont pas aux normes ou sont saturés. Qui va payer ? Les industriels rejettent aujourd'hui une partie de leurs déchets toxiques (traitement des métaux, solvants, peintures...) ... dans les égouts, et bien sur, la mer (baie de Fort de France) accumule !

Pour les ordures ménagères, le taux de couverture du ramassage est satisfaisant depuis plus de dix ans ; le résultat est l'accumulation des déchets sur cinq grandes décharges qui sont bientôt saturées et de toute façon illégales en 2002. Plus de mille dépôts sauvages ont été recensés sur l'île.

Le plan «ordures ménagères » a estimé que sur les 250000 tonnes collectées annuellement, près de la moitié devait faire l'objet d'une incinération avec récupération d'énergie électrique, l'autre moitié pouvant faire l'objet d'un compostage; ce dispositif suppose d'avoir réalisé un tri entre les matières incinérables et les matières compostables.

Ainsi, l'incinérateur de Fort de France, devrait être construit par le SICEM vers 2001/2002 et regroupera les déchets ménagers des quatre grandes communes du Centre : Fort-de-France, Schœlcher, Lamentin et Saint-Joseph. Il entraînera la fermeture de la décharge de la Trompeuse et sa transformation en véritable déchetterie : une déchetterie est un centre de regroupement et de tri à disposition des particuliers; tout citoyen peut y amener son vieux frigo, ses déchets métalliques, des gravats...

L'incinérateur pose plusieurs problèmes techniques qu'il faudra résoudre :

- les déchets incinérés devront être le plus sec possible : donc, les déchets de jardins et espaces verts n'y seront pas admis, mais il faudra également trier les déchets les plus humides, empêcher les eaux de pluie d'humidifier les déchets... 
- l'incinérateur doit être aux normes européennes en ce qui concerne les rejets atmosphériques; en particulier, la concentration en dioxine devra être de 0,1 nanogramme par $\mathrm{m}^{3}$, ce qui impose un surcoût d'investissement important ;

- l'usine d'incinération produira deux types de résidus: l'un inerte, les mâchefers qui peuvent servir de matériau de construction pour les routes, l'autre, très toxique est constitué des fumées et dénommé «REFIOM »; ces résidus à haute toxicité doivent être impérativement stockés dans une décharge spéciale dite de «classe $1 »$.

Aucune décharge de «classe $1 »$ n'existe à la Martinique et les études préliminaires montrent que la création d'un tel site est possible sur un plan technique: il existe à la Martinique des couches d'argile suffisamment épaisses et imperméables; par contre, la rentabilité économique d'un tel dispositif n'est pas démontrée ; la seule alternative reste l'exportation vers la métropole.

Le deuxième équipement prévu est l'usine de compostage qui sera réalisée à la suite de l'incinérateur ; le principe du compostage est simple, mais sa mise en œuvre est soumise à deux conditions :

- le niveau de tri des ordures doit être maximum pour permettre une qualité «agronomique » à un prix attractif pour l'agriculture; les conditions en Martinique sont plutôt favorables puisque l'agriculture consomme uniquement des fertilisants chimiques importés à des prix élevés ;

- l'alternative de fumure organique doit être intéressante au niveau des prix, mais aussi vis-à-vis des pratiques culturales en vigueur; c'est probablement la culture de la canne à sucre et le maraîchage qui seront les principaux débouchés de ce compost.

Une dernière remarque sur une filière très présente en Martinique et dont j'ai peu parlé : les véhicules usagés (VHU); aujourd'hui, seules les huiles usagées sont collectées et on estime à environ $70 \%$ le niveau de récupération en Martinique. Par contre, chaque année, 15000 voitures neuves 
rentrent sur l'île, aucune ne ressort. Chaque voiture a une batterie et les batteries de remplacement entrent également en grande quantité.

Cette filière n'est pas organisée aujourd'hui; les importateurs de batterie ont constitué récemment une association qui étudie avec l'appui de l'ADEME les différentes solutions envisageables; le surcoût lié à l'exportation vers la métropole pour recyclage a été estimé à $5 \mathrm{~F}$ minimum par batteries.

Pour résoudre le problème des carcasses de voitures, la seule solution durable est l'exportation des parties métalliques vers les aciéries de Trinidad ou du Vénézuela, mais cette opération a un coût de $400 \mathrm{~F}$ au moins par carcasse.

\section{EN GUISE DE CONCLUSION}

Je n'ai pas du tout abordé la question de la réhabilitation des décharges.

Je n'ai pas parlé des DTQD «déchets toxiques en quantité diffuse » qui sont en fait les piles que nous utilisons tous : il est de notre responsabilité de pollueur de les collecter, au super marché, chez le photographe...

Je n'ai pas assez parlé des techniques de tri «à la source», chez chacun d'entre nous, techniques qui permettent de séparer les différents sousproduits recyclables : aux USA, des privés proposent des abonnements aux particuliers pour des niveaux de tri variables, mais les boites de conserves, par exemple, doivent être nettoyées de leur étiquette, parfaitement découpées, classées par tailles et matériau !

Nous avons donc du chemin à parcourir et probablement, le déchet restera dans les vingt années à venir un des problèmes majeurs de notre société, donc il vaut mieux essayer d'en parler tout de suite au niveau du citoyen; nous avons individuellement un rôle important à jouer dans la prise de conscience que ce que nous jetons se dégrade de moins en moins : il y a 
quarante ans, notre poubelle contenait plus de la moitié de produits dégradables, aujourd'hui, regardez ce que vous jetez: plus de la moitié en volume est constituée d'emballages, en grande partie en plastiques.

Jean-Louis MILLO

Directeur Régional

de la DRIRE

Martinique 